\title{
CONSENSOS SOBRE LA NATURALEZA DE LA CIENCIA: FUNDAMENTOS DE UNA INVESTIGACIÓN EMPÍRICA
}

\author{
José Antonio Acevedo-Díaz ${ }^{(1)}$, Ángel Vázquez-Alonso( ${ }^{(2)}, M^{a}$ Antonia Manassero-Mas ${ }^{(3)}$ y \\ Pilar Acevedo-Romero ${ }^{(4)}$
}

(1) Inspección de Educación. Consejería de Educación de la Junta de Andalucía.

Delegación Provincial de Huelva. E-mail: ja_acevedo@vodafone.es

(2) Facultad de Ciencias de la Educación. Universidad de las Islas Baleares. E-mail: angel.vazquez@uib.es

(3) Departamento de Psicología. Universidad de las Islas Baleares. E-mail:

ma.manassero@uib.es

(4) IES "Fray Diego Tadeo González", Ciudad Rodrigo (Salamanca). E-mail:

pi_acevedo@yahoo.es

[Recibido en Junio de 2006, aceptado en Septiembre de 2006]

\section{RESUMEN ${ }^{\text {(Inglés) }}$}

Las cuestiones relativas a la naturaleza de la ciencia constituyen hoy uno de los elementos esenciales de la alfabetización científica y tecnológica para todas las personas. Sin embargo, la inclusión de la naturaleza de la ciencia en el currículo de ciencias no es fácil y requiere, entre otras cosas, el establecimiento previo de acuerdos sobre sus principales características y los contenidos que podrían trasladarse al aula para su tratamiento explícito en la educación científica. En este artículo se revisan algunas aportaciones de la didáctica de las ciencias a estas importantes cuestiones. Así mismo, se describe una metodología empírica destinada a conseguir un consenso sobre la naturaleza de la ciencia, entendida ésta en un sentido amplio; acuerdos que se extienden tanto a los aspectos que se consideran adecuados como a lo que se estiman inadecuados.

Palabras clave: Naturaleza de la ciencia, disenso, consenso, currículo de ciencias, Cuestionario de Opiniones CTS, metodología empírica.

\section{INTRODUCCIÓN}

Los currículos de ciencias suelen centrarse sobre todo en los contenidos conceptuales y regirse por la lógica interna de la ciencia, pero se olvidan de dar formación sobre la ciencia misma, es decir, sobre qué es la ciencia, cómo funciona internamente, cómo se desarrolla, cómo construye su conocimiento, cómo se relaciona con la sociedad, qué valores utilizan los científicos en su trabajo profesional, etc. Todos estos aspectos constituyen lo que se conoce como naturaleza de la ciencia ( $\mathrm{NdC}$ en adelante). Como consecuencia de esta falta de atención, la imagen de la ciencia transmitida por la enseñanza tradicional suele estar trasnochada y deformada, pues corresponde a la de 
un conocimiento acabado, definitivo $y$, por ende, autoritario, dogmático e incontestable (Fernández et al., 2002, 2003; Fernández, Gil-Pérez, Valdés y Vilches, 2005; Gil-Pérez et al., 2005). Además, la ciencia escolar tiende a transmitir una imagen de la ciencia del pasado, la que produjo los conceptos habitualmente incluidos en el currículo, pero no de la ciencia y la tecnociencia contemporáneas, las cuales se hacen hoy en los laboratorios de diversas instituciones (universidades, hospitales, fundaciones, ejército, etc.) y empresas privadas (industrias, corporaciones farmacéuticas, etc.).

Para superar estos graves inconvenientes en la actualidad hay un consenso creciente que propone incluir explícitamente en los currículos de ciencias una enseñanza sobre la ciencia misma, es decir, una enseñanza de la NdC. La importancia curricular de la NdC es cada vez más apreciada por quienes diseñan una educación científica para el siglo XXI. Hoy en día diversos países (Australia, Canadá, EE.UU., Nueva Zelanda, Reino Unido, etc.) incluyen explícitamente la enseñanza de la NdC en sus currículos de ciencias reformados (AAAS, 1989, 1993; NRC, 1996; McComas y Olson, 1998, entre otros) y muchos más lo hacen de manera parcial o implícita. En efecto, las diversas reformas de la educación científica resaltan la importancia de desarrollar una imagen de la ciencia más válida desde el punto de vista epistemológico, que sea más coherente con las prácticas científicas actuales (Driver, Leach, Millar y Scott, 1996; Hodson, 1988; Matthews, 1994; Millar y Osborne, 1998; Ryan y Aikenhead, 1992). Desde esta perspectiva, una persona no debería considerarse alfabetizada en ciencia si no es capaz de comprender los valores y supuestos de la actividad científica y los procesos mediante los cuales se crea el conocimiento científico y no llega a superar una imagen ingenua de la ciencia basada en hechos aislados de su contexto, lo que confiere a este conocimiento su relevancia y aplicabilidad (Lederman, 1999). De esta forma, las cuestiones de NdC constituyen uno de los elementos innovadores y destacados de la alfabetización científica y tecnológica para todas las personas y, asimismo, un aspecto esencial y central del currículo de ciencias inspirado por este lema (Acevedo, Manassero y Vázquez, 2002, 2005; Acevedo, Vázquez y Manassero, 2002, 2003; McComas, Clough y Almazroa, 1998; McComas y Olson, 1998; Millar, 1996; Millar y Osborne, 1998; Smith y Scharmann, 1999; Spector, Strong y Laporta, 1998). Ésta es una posición que también ha sido asumida en parte por el proyecto $\mathrm{PISA}^{1}$ (OECD, 2003).

La NdC es un metaconocimiento sobre la ciencia que surge de las reflexiones interdisciplinares realizadas desde la filosofía, la historia y la sociología de la ciencia por especialistas de estas disciplinas y, también, por algunos científicos insignes. En un sentido amplio, la NdC incluye la reflexión respecto a los métodos para validar el conocimiento científico, los valores implicados en las actividades de la ciencia, las relaciones con la tecnología, la naturaleza de la comunidad científica, las relaciones de la sociedad con el sistema tecnocientífico y las aportaciones de éste a la cultura y al

\footnotetext{
${ }^{1}$ PISA es el acrónimo de Programme for International Student Assessment, una evaluación comparativa internacional de carácter trienal promovida por la Organization for Economic Cooperation and Development (OECD en inglés; OCDE en castellano) con el propósito de evaluar el resultado de los sistemas educativos respecto a la formación del alumnado necesaria para la vida adulta. Teniendo en cuenta que la gran mayoría de los estudiantes participantes no serán científicos en el futuro, la evaluación PISA de ciencias da prioridad a los procedimientos relativos al funcionamiento de la ciencia en vez de a los procedimientos que se emplean en la ciencia (véase, p.e., Acevedo, 2005).
} 
progreso de la sociedad. Estas reflexiones interdisciplinares provienen de la controversia y la discusión entre filósofos, historiadores, sociólogos, científicos, profesores y expertos en didáctica de las ciencias (Laudan, 1990; Norris, 1997), y son tan amplias y ricas en matices que pretender resumirlas en unas pocas líneas es una ardua tarea. Las características señaladas apuntan el origen de una de sus principales dificultades para la inclusión de la NdC en la educación científica (Lederman, Wade y Bell, 1998; Vázquez, Acevedo, Manassero y Acevedo, 2001).

La filosofía contemporánea de la ciencia se encuentra hoy en un marco dialéctico y complejo, plagado de propuestas y contrapropuestas, donde a la vez que se intenta dar nuevas respuestas a los sucesivos problemas que se han ido planteando se hacen nuevas críticas a las respuestas anteriores. El resultado es que, entre el positivismo lógico de la primera mitad del siglo $\mathrm{XX}$ y el relativismo constructivista radical del último cuarto del mismo siglo, en la actualidad se pueden encontrar epistemologías de todos los tipos: realismo, neopositivismo, funcionalismo, relativismo y casi cualquier combinación posible de las anteriores, tales como racionalismo crítico, evolucionismo, realismo crítico, realismo constructivo, realismo transformativo, realismo perspectivo, realismo pragmático, empirismo constructivo, constructivismo sociológico, relativismo débil, etc. (Echeverría, 1999; Laudan, 1990, 1996; Vázquez, Acevedo, Manassero y Acevedo, 2001).

En la medida en que la propia empresa científica es poliédrica y dinámica, resulta difícil definir con más precisión el concepto de $\mathrm{NdC}$, aunque se puede decir que, en general, trata de todo aquello que caracteriza a la ciencia como una forma de construir conocimiento (Aikenhead y Fleming, 1975). Además, la NdC también hace referencia a los valores propios y contextuales de la ciencia (Acevedo, 1998; Echeverría, 2002; Longino, 1983, 1990) y a los supuestos subyacentes al conocimiento científico, que son consecuencia del carácter humano de la propia empresa científica, incluyendo sus limitaciones e influencias de todo tipo (Acevedo, 1997, 2006; Collins, 1985; Ziman, 2000, 2003). De este modo, Ryan y Aikenhead (1992) describen la NdC como un conjunto que abarca el significado de la ciencia, supuestos, valores, invenciones conceptuales, métodos, construcción de acuerdos y características epistemológicas propias del conocimiento elaborado.

El concepto de NdC es, pues, dialéctico y complejo, por lo que es comprensible que resulte difícil definirlo con precisión y consenso. Los especialistas debaten a menudo descripciones y representaciones de la NdC que son tan dinámicas como el propio conocimiento científico, de manera que no es posible sostener la existencia de una única NdC capaz de representar a este conocimiento ni a todas las disciplinas científicas. Por consiguiente, cualquier representación de la NdC tenderá a ser parcial y se verá obligada a convivir en competencia con otras representaciones también sesgadas.

Una consecuencia directa de la esencia dialéctica de la noción de NdC es que resulta difícil precisar con exactitud su contenido y, de hecho, la bibliografía especializada utiliza concepciones que no son coincidentes (Bell et al., 2001). La revisión de los principales estudios sobre este tema permite comprobar la existencia de un abanico de interpretaciones, desde la más restringida, donde la NdC se refiere estrictamente a cuestiones epistemológicas sobre la ciencia, hasta otras más amplias que incluyen 
diversos tipos de cuestiones en las que interviene la ciencia, tales como las relaciones mutuas entre ciencia, tecnología y sociedad, la sociología interna de la ciencia, la imagen de la ciencia, las actitudes hacia la ciencia escolar, etc. (Acevedo, Vázquez y Manassero, 2002). Como podrá verse más adelante, en este artículo se sigue el segundo punto de vista.

\section{DIFICULTADES PARA IMPLANTAR LA ENSEÑANZA DE LA NATURALEZA DE LA CIENCIA EN EL AULA}

La ciencia se presenta en la escuela habitualmente como un cuerpo bien establecido de conocimientos acabados y verdaderos que requieren escasa justificación y pocas pruebas, excepto quizás algunas generalizaciones inductivas, con el objeto de persuadir a los estudiantes de la validez de la visión científica del mundo (Ryder, Leach y Driver, 1999). En este escenario, los rasgos formales de la NdC, tales como la provisionalidad del conocimiento científico o sus dimensiones sociales, resultan marginales para el proyecto principal de la ciencia escolar, que suele ser enseñar sobre lo que se sabe pero no sobre como se ha llegado a saberlo (Bartholomew, Osborne y Ratcliffe, 2004; Ogborn, Kress, Martins y McGillicuddy, 1996).

La importancia de la NdC en la educación científica parece que está clara actualmente para la didáctica de las ciencias (Bell et al., 2001), pero los medios para lograr sus propios objetivos de enseñanza todavía no lo están tanto (Bell, 2005). Como elemento curricular central de la alfabetización científica y tecnológica, la enseñanza de la NdC es una innovación en la educación científica de extraordinaria magnitud y no es fácil llevarla a cabo. Como se sabe, los sistemas escolares tienden a generar una serie de resistencias a cualquier innovación, pero la que persigue la inclusión de la NdC en el currículo de ciencias se enfrenta, además, a dos grandes obstáculos específicos:

- La propia NdC como contenido multidisciplinar, dialéctico, complejo y cambiante. No hay precedentes escolares suficientemente convincentes de un currículo de ciencias semejante $y$, además, los rasgos de un currículo innovador de este tipo contrastan con el carácter acabado y dogmático de los contenidos tradicionales de los currículos de ciencias.

- La carencia de formación del profesorado de ciencias para afrontar esta innovación porque, en general, no ha sido preparado para ello, dado que los conocimientos de esta área no suelen formar parte de su formación científica universitaria ni de su formación profesional como docente. En consecuencia, es difícil encontrar entre el profesorado de ciencias una actitud plenamente positiva hacia la inclusión de la $\mathrm{NdC}$ en la ciencia escolar.

Este artículo se centra en el primero de los obstáculos, la complejidad de la NdC y sus dificultades para convertirse en un contenido curricular de la educación científica, sobre el cual la bibliografía especializada muestra dos corrientes de opinión opuestas:

- Una posición de disenso respecto a la NdC que sostiene que no es posible alcanzar acuerdos básicos sobre NdC. El indicador más fiable de esta posición, que ha permanecido implícita durante muchos años, es la exclusión de la NdC de los currículos de ciencias. Así pues, esta perspectiva considera que la NdC no es un 
contenido esencial para la alfabetización científica o, en el mejor de los casos, que su inclusión debería hacerse de una manera muy limitada.

- Una posición de consenso respecto a la $\mathrm{NdC}$ que defiende la posibilidad de lograr algunos acuerdos sobre $\mathrm{NdC}$, a pesar de su complejidad y de los desacuerdos existentes en ciertos temas. Desde esta perspectiva se promueve la inclusión de la $\mathrm{NdC}$ en el currículo de ciencias a partir de los posibles consensos alcanzables, de modo que los estudiantes puedan aprender algo de NdC.

La investigación en didáctica de las ciencias ha dado algunas respuestas parciales, unas favorables y otras desfavorables, al consenso relativo a la NdC para la enseñanza de las ciencias. En los siguientes apartados se revisarán las principales aportaciones de ambas líneas de pensamiento, que servirán como introducción y análisis de los antecedentes de la investigación que se propone en este estudio.

\section{DISENSO RESPECTO A LA NATURALEZA DE LA CIENCIA}

La reflexión histórica, sociológica y filosófica sobre la ciencia plantea la descripción y explicación de la actividad científica y la validación del conocimiento mediante el ejercicio de una crítica permanente de las diferentes ideas e interpretaciones aportadas. Esta metodología proporciona una imagen de la $\mathrm{NdC}$ como un área de disenso y litigio, que produce controversias poco comprensibles para los no iniciados, debilita el compromiso con la validación de sus propias afirmaciones y dificulta la creación de propuestas interesantes para la propia ciencia y, a menudo, también para su didáctica y educación. Las escasas posibilidades de consensos, que podrían servir de base para la inclusión de la NdC en el currículo ciencias, destacan por su fragilidad y brevedad, porque la necesidad de nuevas críticas para alimentar la dinámica propia de su metodología diluye las posibilidades de acuerdo en un océano de críticas constantes. De este modo, se impide la emergencia de cualquier representación de la ciencia capaz de superar las críticas y susceptible de ser aceptada mayoritariamente por su valor (Laudan, 1990).

En los años noventa del siglo XX, como reacción a los excesos postmodernos de la denominada "izquierda académica" (representada sobre todo por relativistas y socialconstructivistas radicales), el impacto causado por un libro de Gross y Levitt (1994) y la publicación como artículo de una broma pesada (Sokal, 1996) dieron lugar a un enconado enfrentamiento entre las comunidades académicas de "letras" y "ciencias", que se conoce con el llamativo nombre de "guerra de las ciencias". Esta polémica muestra de manera extrema el disenso correspondiente a la NdC entre científicos y filósofos o sociólogos de la ciencia, y ha puesto en escena el rechazo frontal por parte de la mayoría de los científicos de las afirmaciones postmodernas más radicales sobre la NdC (Wolpert, 1992; Sokal y Bricmont, 1998). Los debates de la "guerra de las ciencias" promueven discusiones de un nivel relativamente elevado, en las que, además, las divergencias tienen demasiada carga demagógica y se encuentran aparentemente muy lejos de la educación científica, pero que no dejan de afectar a la enseñanza de la NdC.

El estudio empírico de Alters (1997), basado en una encuesta con 11 proposiciones que se aplicó a 210 filósofos, es quizás el más representativo de la línea de disenso 
respecto a la NdC desde el punto de vista de la didáctica de las ciencias. En ese trabajo, el autor muestra la existencia de profundos desacuerdos entre los filósofos, incluso en los temas más básicos de epistemología de la ciencia y, por tanto, concluye que la enseñanza de la NdC debería realizarse desde una perspectiva de mayor pluralismo epistemológico, que evite el adoctrinamiento. No obstante, este trabajo ha sido muy contestado, pues la revisión de los propios datos aportados permite identificar acuerdos restringidos, pero apreciables, en algunos asuntos de NdC (Smith et al., 1997); una conclusión que es contraria al desacuerdo que sostiene su autor. A Alters también se le reprocha haber obtenido un disenso artificial, inducido por una encuesta que parece sesgada hacia los temas más polémicos de la NdC y por usar una muestra homogénea, exclusivamente constituida por filósofos, debido a la tendencia profesional de éstos a disentir más que a establecer consensos. Osborne et al. (2003) han sugerido al respecto la posibilidad de haber alcanzado importantes acuerdos si el mismo cuestionario se hubiera aplicado a otro grupo de profesionales diferentes como, por ejemplo, científicos especialistas en física.

En definitiva, aunque la complejidad dialéctica y el disenso son una realidad en el ámbito de la $\mathrm{NdC}$, las críticas anteriores también permiten vislumbrar la posibilidad de conseguir acuerdos, de modo que el mayor interés de la investigación en didáctica de las ciencias parece que debe centrarse en identificar esos consensos potenciales.

\section{CONSENSO RESPECTO A LA NATURALEZA DE LA CIENCIA}

Desde hace décadas, la investigación de las denominadas actitudes hacia la ciencia y actitudes científicas han sido la principal fuente de referencia sobre la existencia de acuerdos parciales relativos a la NdC, si bien estos estudios no estaban directamente dirigidos a la búsqueda de un consenso respecto a la NdC. Estos acuerdos son claramente visibles, por ejemplo, en la estructura y contenidos de muchos de los instrumentos empleados para la evaluación de actitudes en torno a una serie de características de la ciencia, tales como su carácter empírico, el uso prioritario de la lógica, la falibilidad, la creatividad, etc. (Bell et al., 2001; Lederman, 1992; Vázquez y Manassero, 1995). Posteriormente, el enfoque CTS (acrónimo de Ciencia, Tecnología y Sociedad), como orientación de la enseñanza de las ciencias, permitió dar fundamento a las primeras propuestas innovadoras con una significativa presencia de asuntos relacionados con la $\mathrm{NdC}$, tomados en un sentido amplio y no restringido a lo puramente epistemológico (Acevedo, Manassero y Vázquez, 2002, 2005; Acevedo, Vázquez y Manassero, 2002, 2003; Aikenhead, 2003).

Como se ha indicado en la sección anterior, una prueba a favor del consenso sobre NdC proviene de la atinada crítica de Smith et al. (1997) al estudio de Alters (1997) favorable a la tesis del disenso. En otra crítica a ese estudio, Eflin, Glennan y Reisch (1999) resumen algunos de los principales acuerdos y desacuerdos acerca de la NdC entre los expertos en educación científica. Según estos autores, el consenso se puede centrar en que (i) el principal propósito de la ciencia es adquirir conocimiento sobre el mundo físico, (ii) en el mundo hay un orden fundamental que la ciencia pretende describir de la manera más simple y comprensiva posible, (iii) la ciencia es cambiante, dinámica y provisional, y (iv) no existe un único método científico. Por el contrario, los desacuerdos más importantes aparecen en aspectos como (i) la generación del 
conocimiento científico depende de compromisos teóricos y factores contextuales sociales e históricos (contextualismo), y (ii) la verdad de las teorías científicas viene determinada por aspectos del mundo que existen de modo independiente de los científicos (realismo ontológico). Los autores consideran que casi todos los filósofos de la ciencia y los propios científicos están de acuerdo con los especialistas en didáctica de las ciencias en los puntos de consenso señalados. Así mismo, sugieren que aquellos aspectos donde no hay acuerdo entre los expertos en educación científica también son bastante controvertidos para los filósofos de la ciencia (Eflin, Glennan y Reisch, 1999).

En esta línea favorable al consenso, diversos trabajos que muestran acuerdos relativos a la NdC en la educación científica comenzaron a aparecer en la década de los noventa. Por ejemplo, Rubba y Harkness (1993) desarrollaron un conjunto de diez cuestiones de opción múltiple -el TBA-STS (Teacher's Belief about ScienceTechnology-Society) - para investigar las creencias del profesorado de ciencias sobre las relaciones CTS. Las frases de las opciones múltiples de esas cuestiones fueron valoradas por cinco científicos que actuaron como jueces expertos. Las afirmaciones clasificadas como "realistic" (tabla 1), cuyo contenido se corresponde con una idea adecuada a los conocimientos contemporáneos de historia, filosofía y sociología de la ciencia, muestran un conjunto de acuerdos sobre NdC (Rubba, Schoneweg-Bradford y Harkness, 1996). Así mismo, la construcción del cuestionario Views on the Nature of Science -VNOS- (Lederman, Abd-El-Khalick, Bell y Schwartz, 2002) se basó en un conjunto de características concretas de la NdC que articularían el consenso respecto a la NdC (tabla 2).

El análisis de los currículos de ciencias también puede ser una fuente del consenso correspondiente a la NdC (Felske, Chiappetta y Kemper, 2001). El estudio transversal de ocho documentos curriculares internacionales (McComas, Clough y Almazroa, 1998) muestra los acuerdos sobre NdC alcanzados en la educación científica de diferentes países. Los documentos propuestos por grupos de diseño curricular constituidos por expertos heterogéneos, que actuaban de modo independiente en varios países y con distintos criterios, permitieron a los autores del estudio identificar 16 propuestas de consenso (tabla 3), obtenidas a partir de las coincidencias en los contenidos de todos los documentos analizados por McComas y Olson (1998).

Estos contenidos curriculares de NdC podrían interpretarse como indicadores de cierto grado consenso respecto a la NdC, por encima de las discrepancias existentes; de otra forma, parecen mostrar una firme creencia en la necesidad y posibilidad de enseñar $\mathrm{NdC}$ en la ciencia escolar. Sin embargo, como acertadamente observan Osborne et al. (2003), los comités para la redacción de estos currículos no ofrecen suficiente garantía de haber alcanzado un consenso basado en pruebas empíricas que puedan servir para su validación. De hecho, algunos aspectos de ellos han suscitado críticas y provocado redacciones diferentes en ediciones sucesivas -por ejemplo, el Project 2061 de la AAAS (1989), los Benchmarks de la AAAS (1993) y los Standards del National Research Council (NRC, 1996) en EE.UU.-, lo que hace sospechar que las decisiones curriculares sean meros compromisos internos de los propios comités para salir adelante en su tarea. Por tanto, aunque los currículos de ciencias publicados suponen un cierto avance, quedaría aún pendiente la tarea de dar mejor fundamento a los acuerdos potenciales respecto a la NdC. 
10111 Definir qué es la ciencia es difícil porque ésta es algo compleja y engloba muchas cosas. Pero la ciencia PRINCIPALMENTE es:

H. Un proceso investigador sistemático y el conocimiento resultante.

$10113 \mathrm{El}$ proceso de hacer ciencia se describe mejor como... :

F. Observar y proponer explicaciones sobre las relaciones en el universo y comprobar la validez de las explicaciones.

10211 Definir qué es la tecnología puede resultar difícil porque ésta sirve para muchas cosas. Pero la tecnología PRINCIPALMENTE es:

G. Ideas y técnicas para diseñar y hacer cosas, para organizar a los trabajadores, la gente de negocios y los consumidores, y para el progreso de la sociedad.

10412 ¿La ciencia influye en la tecnología?

E. La ciencia es el conocimiento base para la tecnología.

10413 ¿La tecnología influye en la ciencia?

C. La disponibilidad de tecnología influye en la dirección de la investigación científica.

F. La tecnología suministra herramientas y técnicas para la ciencia.

20811 ¿La sociedad influye en la tecnología?

D. La sociedad vota a favor o en contra de ciertas tecnologías cada vez que compramos algo.

F. La sociedad crea demandas a la tecnología y las restringe basándose en los valores de lo que es importante para mejorar la vida.

20821 ¿La sociedad influye en la ciencia?

D. La sociedad determina qué tipo de investigación científica es aceptable, basándose en nuestros valores morales y éticos.

F. La sociedad influye en la ciencia a través de las subvenciones económicas de las que dependen la mayoría de las investigaciones.

40811 ¿La tecnología influye en la sociedad?

C. La tecnología forma parte de todos los aspectos de nuestras vidas, desde el nacimiento hasta la muerte.

D. La tecnología influye en la sociedad mediante la manera en que ésta la utiliza.

E. La tecnología proporciona a la sociedad los medios para mejorar o destruirse a sí misma, dependiendo de como se ponga en práctica.

F. La sociedad cambia como resultado de aceptar una tecnología.

40821 ¿La ciencia influye en la sociedad?

D. La ciencia capacita a las personas para poder conocer el mundo.

90511 Las ideas científicas se desarrollan desde las hipótesis hasta las teorías y, finalmente, si son suficientemente buenas, hasta constituir leyes.

Las hipótesis pueden conducir a teorías que pueden llevar a leyes:

E. Las teorías no pueden convertirse en leyes porque ambas son ideas de distinta clase. Las leyes describen fenómenos naturales. Las teorías explican fenómenos naturales. Por tanto, las teorías no pueden convertirse en leyes. Sin embargo, con pruebas que las apoyen, las hipótesis pueden convertirse en teorías (explicaciones) o leyes (descripciones).

Tabla 1.- Afirmaciones de NdC consideradas adecuadas ("realistic") por Rubba, Schoneweg-Bradford y Harkness (1996). 


\begin{tabular}{|c|c|}
\hline Provisionalidad & $\begin{array}{l}\text { El conocimiento científico está sujeto a cambios, con nuevas observaciones } \\
\text { y reinterpretaciones de las observaciones existentes. Todos los demás } \\
\text { aspectos de la NdC apoyan la provisionalidad del conocimiento científico. }\end{array}$ \\
\hline Base empírica & $\begin{array}{l}\text { El conocimiento científico se basa o se deriva de las observaciones del } \\
\text { mundo natural. }\end{array}$ \\
\hline Subjetividad & $\begin{array}{l}\text { La ciencia está influida y guiada por las teorías científicas y las leyes } \\
\text { aceptadas. La formulación de preguntas, las investigaciones y las } \\
\text { interpretaciones de los datos se filtran a través de la teoría vigente. Ésta es } \\
\text { una subjetividad inevitable, pero le permite a la ciencia progresar y } \\
\text { permanecer consistente. El examen de las pruebas anteriores desde la } \\
\text { perspectiva del nuevo conocimiento también contribuye al cambio en la } \\
\text { ciencia. }\end{array}$ \\
\hline Personal & $\begin{array}{l}\text { La subjetividad personal también es inevitable. Los valores personales, las } \\
\text { prioridades y las experiencias anteriores dictan hacia donde y cómo los } \\
\text { científicos dirigen su trabajo. }\end{array}$ \\
\hline Creatividad & $\begin{array}{l}\text { El conocimiento científico se genera mediante la imaginación humana y el } \\
\text { razonamiento lógico. Esta creación se basa en observaciones del mundo } \\
\text { natural y en las inferencias que se hacen. }\end{array}$ \\
\hline $\begin{array}{l}\text { Inmersión } \\
\text { Sociocultural }\end{array}$ & $\begin{array}{l}\text { La ciencia es un empeño humano y está influida por la sociedad y la cultura } \\
\text { donde se desarrolla. Los valores de la cultura determinan hacia donde se } \\
\text { dirige la ciencia, cómo lo hace, se interpreta, se acepta y se utiliza. }\end{array}$ \\
\hline $\begin{array}{l}\text { Observación e } \\
\text { inferencia }\end{array}$ & $\begin{array}{l}\text { La ciencia se basa en la observación y la inferencia. Las observaciones se } \\
\text { recogen por medio de los sentidos humanos o extensiones de éstos. Las } \\
\text { inferencias son interpretaciones de esas observaciones. Las perspectivas } \\
\text { actuales de la ciencia y los científicos guían las observaciones e inferencias. } \\
\text { Diversas perspectivas contribuyen a múltiples interpretaciones válidas de } \\
\text { las observaciones. }\end{array}$ \\
\hline Leyes y teorías & $\begin{array}{l}\text { Las teorías y las leyes son diferentes tipos de conocimiento científico. Las } \\
\text { leyes describen las relaciones, observadas o percibidas, de los fenómenos } \\
\text { de la naturaleza. Las teorías son explicaciones inferidas de los fenómenos } \\
\text { naturales y los mecanismos de las relaciones entre estos fenómenos } \\
\text { naturales. Las hipótesis científicas pueden conducir a teorías o a leyes } \\
\text { mediante la acumulación de apoyo sustancial con pruebas y la aceptación } \\
\text { por la comunidad científica. Las teorías y las leyes no se convierten unas en } \\
\text { otras, en sentido jerárquico, porque ambas son tipos de conocimiento } \\
\text { explícita y funcionalmente diferentes. }\end{array}$ \\
\hline $\begin{array}{l}\text { Interdependencia } \\
\text { de los rasgos de la } \\
\text { ciencia }\end{array}$ & $\begin{array}{l}\text { Ninguno de los aspectos anteriores puede considerarse separado de los } \\
\text { demás. Por ejemplo, la provisionalidad del conocimiento científico proviene } \\
\text { de la creación de ese conocimiento mediante la observación empírica y la } \\
\text { inferencia. Cada una de estas actividades está influida por la cultura y la } \\
\text { sociedad donde se practica la ciencia, así como por el marco conceptual y la } \\
\text { subjetividad personal de cada científico. Cuando se consideran nuevos } \\
\text { datos y se revisan los existentes, las inferencias (realizadas en un contexto } \\
\text { particular) pueden llevar a cambios en el conocimiento científico existente. }\end{array}$ \\
\hline
\end{tabular}

Tabla 2.- Propuestas de consenso respecto a la NdC tomadas del proceso de construcción del cuestionario Views on the Nature of Science (VNOS). 
$\checkmark$ Aunque es duradero, el conocimiento científico tiene carácter provisional.

$\checkmark$ El conocimiento científico se basa fundamentalmente, pero no por completo, en observación, pruebas empíricas, argumentos racionales y escepticismo.

$\checkmark$ No existe una sola manera de hacer ciencia; por tanto, no hay ningún método científico universal en etapas sucesivas.

$\checkmark \quad$ La ciencia es un esfuerzo para explicar los fenómenos naturales.

$\checkmark$ Las leyes y teorías desempeñan papeles diferentes en la ciencia; las teorías no se convierten en leyes acumulando más pruebas adicionales.

$\sqrt{ } \quad$ Las personas de todas las culturas contribuyen a la ciencia.

$\checkmark$ El conocimiento nuevo debe comunicarse clara y abiertamente.

$\checkmark$ Los científicos necesitan disponer de registros exactos, someterse a la revisión por iguales, información veraz y la posibilidad de replicar los resultados.

Las observaciones científicas están cargadas de teoría.

Los científicos son creativos.

$\checkmark$ La historia de la ciencia revela a la vez un carácter evolutivo y revolucionario.

$\checkmark$ La ciencia es parte de las tradiciones sociales y culturales.

$\checkmark \quad$ La ciencia y la tecnología interactúan entre sí.

$\checkmark \quad$ Las ideas científicas están influidas por su entorno histórico y social.

$\sqrt{ }$ La ciencia tiene implicaciones globales.

$\checkmark \quad$ Los científicos toman decisiones éticas.

Tabla 3.- Afirmaciones que representarían un consenso sobre $\mathrm{NdC}$ interpretado a partir de diversos documentos curriculares (McComas, Clough y Almazroa, 1998) ${ }^{2}$.

Como respuesta a ello, Osborne et al. (2003) han establecido empíricamente un posible consenso acerca de las "ideas sobre la ciencia" que deberían enseñarse a los estudiantes, mediante la aplicación de una metodología Delphi desarrollada en tres etapas y en la que participaron 23 expertos. Después de las tres etapas del estudio, aplicando criterios muy exigentes para el consenso, basados en el acuerdo de dos tercios de los expertos con puntuaciones iguales o superiores a 4 en una escala de 5 puntos y la estabilidad de los resultados obtenidos en las diferentes etapas de la metodología aplicada, se decantó un conjunto de ideas clave agrupadas en diez temas que los autores proponen como base de los contenidos de $\mathrm{NdC}$ aptos para el currículo de ciencias (tabla 4). La serie de acuerdos establecidos se centra prácticamente en cuestiones de epistemología de la ciencia, con una pequeña concesión a la historia y la sociología interna de la ciencia en el tema denominado "cooperación y colaboración en el desarrollo del conocimiento científico".

\footnotetext{
2 Se han subrayado las características que están presentes en seis o más de los ocho documentos internacionales analizados por McComas y Olson (1998).
} 


\begin{tabular}{|c|c|}
\hline TEMAS & $\begin{array}{c}\text { Recomendaciones para el currículo. Debería enseñarse } \\
\text { que ... }\end{array}$ \\
\hline Ciencia y curiosidad & $\begin{array}{l}\text { Un aspecto importante del trabajo científico es el continuo proceso } \\
\text { cíclico de hacer preguntas y buscar respuestas que conducen a nuevas } \\
\text { preguntas. Este proceso hace emerger nuevas teorías y técnicas } \\
\text { científicas que se prueban empíricamente. }\end{array}$ \\
\hline Creatividad & $\begin{array}{l}\text { La ciencia es una actividad que implica creatividad e imaginación, } \\
\text { como sucede en tantas otras actividades humanas, y algunas ideas } \\
\text { científicas son extraordinarios logros intelectuales. Los científicos, lo } \\
\text { mismo que otros profesionales, son humanos, apasionados y están } \\
\text { comprometidos en su trabajo. También confían en la inspiración y la } \\
\text { imaginación. }\end{array}$ \\
\hline Hipótesis y predicción & $\begin{array}{l}\text { Los científicos formulan hipótesis y hacen predicciones de los } \\
\text { fenómenos naturales. Este proceso es esencial para el desarrollo de } \\
\text { nuevos conocimientos. }\end{array}$ \\
\hline $\begin{array}{l}\text { Métodos científicos y } \\
\text { comprobación crítica }\end{array}$ & $\begin{array}{l}\text { La ciencia usa un método experimental para probar las ideas y, en } \\
\text { particular, ciertas técnicas básicas como el control de variables. } \\
\text { Además, el resultado de un solo experimento pocas veces es suficiente } \\
\text { para establecer un nuevo conocimiento. }\end{array}$ \\
\hline $\begin{array}{l}\text { Análisis e interpretación } \\
\text { de datos }\end{array}$ & $\begin{array}{l}\text { La práctica científica implica destrezas en el análisis e interpretación } \\
\text { de los datos. Los conocimientos científicos no surgen simplemente de } \\
\text { los datos, sino después de un proceso de interpretaciones y } \\
\text { construcción de teorías, lo que requiere habilidades sofisticadas. } \\
\text { También es posible y legítimo que los científicos den diferentes } \\
\text { interpretaciones de los mismos datos y que, por tanto, discrepen. }\end{array}$ \\
\hline $\begin{array}{l}\text { Diversidad del } \\
\text { pensamiento científico }\end{array}$ & $\begin{array}{l}\text { La ciencia utiliza una serie de métodos y enfoques. No existe un único } \\
\text { método científico. }\end{array}$ \\
\hline Ciencia y certeza & $\begin{array}{l}\text { Gran parte del conocimiento científico, sobre todo en la ciencia } \\
\text { escolar, está bien establecido y fuera de toda duda razonable, pero } \\
\text { otra parte del mismo es más dudosa. El conocimiento científico actual } \\
\text { es el mejor que tenemos, pero puede estar sujeto a cambio en el } \\
\text { futuro ante nuevas pruebas o nuevas interpretaciones de las antiguas. }\end{array}$ \\
\hline $\begin{array}{l}\text { Desarrollo histórico del } \\
\text { conocimiento científico }\end{array}$ & $\begin{array}{l}\text { Es necesario conocer un poco de historia del desarrollo del } \\
\text { conocimiento científico. }\end{array}$ \\
\hline $\begin{array}{l}\text { Dimensiones morales y } \\
\text { éticas del desarrollo del } \\
\text { conocimiento científico }\end{array}$ & $\begin{array}{l}\text { Las decisiones en la aplicación del conocimiento científico y técnico no } \\
\text { son neutrales; por tanto, podrían entrar en conflicto con valores } \\
\text { morales y éticos de diversos grupos sociales. }\end{array}$ \\
\hline $\begin{array}{l}\text { Cooperación y } \\
\text { colaboración en el } \\
\text { desarrollo del } \\
\text { conocimiento científico }\end{array}$ & $\begin{array}{l}\text { El trabajo científico es una actividad colectiva y, a la vez, competitiva. } \\
\text { Aunque algunos individuos pueden hacer contribuciones significativas, } \\
\text { el trabajo científico se lleva a cabo con mucha más frecuencia en } \\
\text { grupo, a menudo con carácter multidisciplinar e internacional. } \\
\text { Generalmente, los nuevos conocimientos se comparten y deben } \\
\text { superar un proceso de revisión crítica por los colegas para que sean } \\
\text { aceptados por la comunidad científica. }\end{array}$ \\
\hline
\end{tabular}

Tabla 4.- Ideas sobre la ciencia que consiguen el consenso y deberían enseñarse en la ciencia escolar (Osborne et al., 2003).

La comparación de los consensos establecidos por McComas y Olson (1998) con los obtenidos por Osborne et al. (2003) pone claramente de manifiesto que las relaciones CTS no se consideran en el segundo caso. Por ejemplo, las cuestiones "la ciencia tiene implicaciones globales", "los científicos toman decisiones éticas" y "las personas de todas las culturas contribuyen a la ciencia", citados en el primer estudio, no tienen 
equivalentes en el segundo. Así mismo, el tema CTS "la ciencia forma parte de las tradiciones culturales y sociales" señalado por McComas y Olson (1998), con un contenido social claramente externo a la ciencia, se equipara de modo sorprendente en el trabajo de Osborne et al. (2003, p. 713) al tema "cooperación y colaboración en el desarrollo del conocimiento científico", cuyo sentido es más bien interno al sistema tecnocientífico, a juzgar por las ideas incluidas en él. Las relaciones de la ciencia con la sociedad o con la tecnología también son una ausencia muy notable en los acuerdos establecidos en ese trabajo, como los propios autores reconocen. Esta omisión no es debida al olvido de los autores en el planteamiento inicial de la investigación, sino a su eliminación en el proceso de selección al aplicar los criterios establecidos para lograr el consenso. Sin embargo, unas ideas sobre la ciencia desprovistas de las relaciones de ésta con la tecnología y con la sociedad podrían dar una imagen tal vez muy segura, pero ciertamente incompleta de la ciencia y la tecnociencia contemporáneas (Rudolph, 2003).

En suma, la existencia de un consenso respecto a la NdC es una idea cada vez más extendida y compartida entre los especialistas de esta área. En este sentido, cabe resaltar que el trabajo de Bartholomew, Osborne y Ratcliffe (2004) se inicia dando por supuesto que "hay un acuerdo general acerca de que los componentes de la naturaleza de la ciencia son un elemento esencial y central del currículo de ciencias" y, aunque pueda ser sólo una anécdota, esta frase muestra un punto de vista optimista a favor de la aceptación de la idea del consenso relativo a la NdC.

La identificación de consensos positivos de las características que tiene la NdC es una línea de trabajo preponderante porque responde a un enfoque asertivo, pero el conocimiento de la imagen negativa de la NdC es la otra cara de la misma moneda y también ofrece algunas ventajas de interés. En efecto, desde la perspectiva de la didáctica de las ciencias, tan importante como afirmar lo que son las cosas, lo es también decir lo que no son o no pueden ser, porque ayuda a completar la imagen y, sobre todo, a enfrentarse explícitamente a las concepciones alternativas en el currículo de ciencias. Éste sería el caso de los acuerdos acerca de las creencias inadecuadas de NdC. Así pues, otra forma de poner en evidencia el consenso sobre NdC es resaltando los acuerdos existentes respecto a los puntos de vista inadecuados acerca de la NdC; es decir, el consenso en aquellos aspectos que no se pueden aceptar como rasgos propios y característicos de la ciencia.

Las críticas a la visión positivista de la ciencia han puesto de manifiesto ciertos aspectos inadecuados de la NdC que han generado un amplio consenso. McComas $(1996,1998)$ los denomina mitos sobre la NdC, resaltando su carácter de creencias de sentido común que existen en el imaginario personal y colectivo aunque no puedan ser justificadas (tabla 5 ).

Otros autores llaman "visiones deformadas" a las creencias ingenuas sobre la ciencia (Fernández et al., 2002, 2003; Fernández, Gil-Pérez, Valdés y Vilches, 2005; Gil-Pérez et al., 2005), porque crean una imagen de la ciencia distorsionada e inadecuada al carecer de sintonía con la realidad. La explicación de estas visiones deformadas combina muchos de los mitos expresados por McComas (1996, 1998), aunque englobadas en categorías más amplias que a veces se solapan entre ellas. 
$\checkmark \quad$ Las hipótesis se convierten en teorías, las cuales a su vez llegan a ser leyes.

$\checkmark$ Las leyes científicas y otras ideas similares son absolutas.

$\checkmark$ Una hipótesis es una conjetura educada.

$\checkmark$ Existe un método científico general y universal.

$\checkmark$ Las pruebas cuidadosamente acumuladas producirán un conocimiento cierto.

$\checkmark$ La ciencia y sus métodos ofrecen pruebas absolutas.

$\checkmark$ La ciencia es más procesual que creativa.

$\checkmark \quad$ La ciencia y sus métodos pueden resolver todos los problemas.

$\checkmark \quad$ Los científicos son especialmente objetivos.

$\checkmark$ Los experimentos son el principal camino hacia el conocimiento.

$\checkmark$ Las conclusiones científicas son revisadas por precisión.

$\checkmark$ La aceptación de nuevos conocimientos científicos es inmediata.

$\checkmark$ Los modelos de la ciencia representan la realidad.

$\checkmark$ Ciencia y tecnología son la misma cosa.

$\checkmark \quad$ La ciencia es un empeño individual.

Tabla 5.- Mitos acerca de la NdC (McComas, 1996, 1998).

En lo que sigue, se expondrán los principales aspectos metodológicos de una investigación empírica destinada a establecer un consenso sobre la NdC, entendida ésta en el sentido amplio que proporciona el marco teórico del movimiento CTS para la enseñanza de las ciencias (Acevedo, Vázquez y Manassero, 2002); acuerdos que se extienden tanto a los aspectos de la NdC que se consideran adecuados como a lo que se estiman inadecuados.

\section{METODOLOGÍA}

En esta sección se describen los métodos de investigación aplicados para identificar los consensos relativos a la NdC. En primer lugar, la selección de la muestra de expertos que han actuado como jueces para evaluar las frases del cuestionario, en segundo lugar el cuestionario usado como instrumento en la investigación y, por último, los procedimientos y criterios que se aplican en la investigación.

\section{Muestra}

El método elegido para establecer los posibles acuerdos sobre NdC se basa en recoger los juicios de un panel de expertos mediante una encuesta directa que valora cuestiones problemáticas concretas de NdC desde una perspectiva CTS. Los expertos actúan como jueces con distintas opiniones que se sopesan para obtener los acuerdos más sobresalientes y la opinión mayoritaria más valorada. El método de jueces ha sido aplicado en distintos campos para clasificar afirmaciones diversas sobre un tema especialmente controvertido (Eagly y Chaiken, 1993). Este recurso a los jueces 
pretende establecer el juicio más valioso sobre una cuestión en una comunidad de expertos y permite dar cuenta de la magnitud del consenso sobre semejante juicio.

Un factor clave de esta técnica es establecer el número de jueces más adecuado para optimizar la fiabilidad del juicio a partir de sus valoraciones individuales. No existe una norma clara sobre el número más idóneo, pero un mayor tamaño de la muestra suele repercutir en la mayor fiabilidad de los resultados, aunque por encima de cierto número de jueces ya no se consiguen mejoras significativas (Manassero, Vázquez y Acevedo, 2001). No obstante, en cualquier caso, debe garantizarse un número mínimo de jueces para evitar posibles sesgos. Ahora bien, la tarea de juzgar cuestiones tan complejas como las de NdC tiene otras dificultades que se relacionan más con la pericia de los jueces en el tema a valorar que con el número de ellos. Como la población disponible de expertos potenciales no es muy amplia, debido a que la pericia en NdC no es fácil de alcanzar, la muestra de jueces tampoco puede ser muy grande (Rubba y Harkness, 1993; Rubba, Schoneweg-Bradford y Harkness, 1996).

En este contexto, el principal criterio para la selección de candidatos al panel de jueces es que reúnan el perfil de una persona con una especialidad reconocida en la investigación, la enseñanza o el uso de temas de NdC. Para satisfacer este criterio se localizó una serie de personas cuyo interés por el área de NdC estuviera probado por sus publicaciones, participaciones en congresos, actividades profesionales, etc. Inicialmente, se intentó conseguir una muestra de una veintena de expertos, para lo cual se estableció contacto con 26 especialistas que tenían las características señaladas. La variedad de antecedentes profesionales de los expertos con los que se contactó -investigadores en didáctica de las ciencias, filósofos de la ciencia, profesores y asesores de ciencias- garantiza una diversidad de puntos de vista respecto a la temática planteada, que debería traducirse en la interdisciplinariedad característica de esta área mediante la pluralidad de juicios emitidos. Por diversas razones, como no responder a la invitación, rehusar a participar o no completar totalmente el trabajo propuesto, la muestra definitiva de la investigación es de 16 jueces. Todos cumplen la condición de compartir en mayor o menor grado una cierta especialidad en $\mathrm{NdC}$, junto con otra ocupación principal como asesores o formadores de profesores de ciencias (5), filósofos (4), investigadores en didáctica de las ciencias (4) y profesores de ciencias (3). La muestra está compuesta por 5 mujeres y 11 hombres. Cuatro jueces son licenciados en filosofía, uno de ellos a la vez también lo es en ciencias, mientras que los demás (12) son licenciados en ciencias (física, química, biología y geología). Los jueces ejercen como profesores de secundaria (5), asesores de ciencias en centros de formación del profesorado (4) y profesores de universidad e investigadores (7). La mayoría (12) tienen una actividad investigadora reconocida en el ámbito de la didáctica de las ciencias o en la educación CTS.

\section{Instrumento}

Para obtener las opiniones de los jueces sobre NdC se ha empleado como instrumento el Cuestionario de Opiniones sobre CTS (COCTS), constituido por 100 cuestiones de opción múltiple (Manassero, Vázquez y Acevedo, 2001, 2003), que contienen un total de 637 frases en las que se reflejan distintas creencias y actitudes sobre NdC desde una perspectiva CTS amplia que no se limita a lo epistemológico. El COCTS es 
básicamente una adaptación al contexto español de las cuestiones del Views on Science-Technology-Society -VOSTS- (Aikenhead y Ryan, 1989, 1992; Aikenhead, Ryan y Fleming, 1989) y del Teacher's Belief about Science-Technology-Society -TBASTS- (Rubba y Harkness, 1993; Rubba, Schoneweg-Bradford y Harkness, 1996).

TEMAS

\section{SUBTEMAS}

\section{DEFINICIONES DE CIENCIA Y TECNOLOGÍA}

1. Ciencia y Tecnología 01. Ciencia; 02. Tecnología; 03. I+D; 04. Interdependencia.

\section{SOCIOLOGÍA EXTERNA DE LA CIENCIA}

2. Influencia de la Sociedad sobre la Ciencia/Tecnología 01. Gobierno; 02. Industria; 03. Ejército; 04. Ética; 05. Instituciones educativas; 06. Grupos de presión; 07. Influencia sobre los científicos;

3. Influencia triádica CTS 08. Influencia general.

4. Influencia de la Ciencia/Tecnología sobre la Sociedad

01. Interacciones entre ciencia, tecnología y sociedad.

01. Responsabilidad social; 02. Decisiones sociales; 03. Problemas sociales; 04. Resolución de problemas; 05. Bienestar económico; 06. Contribución al poder militar; 07. Contribución al pensamiento social; 08. Influencia general.

5. Influencia de la ciencia escolar sobre la Sociedad

01. Unión de las dos culturas; 02. Fortalecimiento social; 03. Caracterización escolar de la ciencia.

\section{SOCIOLOGÍA INTERNA DE LA CIENCIA}

6. Características de los científicos

7. Construcción social del conocimiento científico

8. Construcción social de la Tecnología

01

01. Motivaciones; 02. Valores y normas; 03. Ideologías; 04. Capacidades; 05. Efectos del género; 06. Insuficiente representación de las mujeres.

01. Colectivización; 02. Decisiones científicas; 03. Comunicación profesional; 04. Competencia profesional; 05. Interacciones sociales; 06. Influencia de los individuos; 07. Influencia nacional; 08. Ciencia pública vs. ciencia privada.

01. Decisiones tecnológicas; 02. Tecnología autónoma.

\section{EPISTEMOLOGÍA}

01. Observaciones; 02. Modelos científicos; 03. Esquemas de

9. Naturaleza del conocimiento científico clasificación; 04. Provisionalidad; 05. Hipótesis, teorías y leyes; 06. Aproximación a las investigaciones; 07. Precisión e incertidumbre; 08. Razonamiento lógico; 09. Supuestos de la ciencia; 10 . Estatus epistemológico; 11 . Paradigmas y coherencia de conceptos.

Tabla 6.- Contenidos de las relaciones entre ciencia, tecnología y sociedad (CTS) correspondientes a la NdC. 
Las cuestiones del COCTS se encuentran agrupadas en las dimensiones, temas y subtemas que se indican en la tabla 6 . Dentro de cada subtema hay un número variable de cuestiones. Todas las cuestiones tienen el mismo formato de respuesta múltiple, que se inicia con una cabecera de pocas líneas donde se plantea un problema, seguido de una lista de frases que ofrecen un abanico de diferentes respuestas razonadas al problema planteado, entre 6 y 7 frases de promedio por cuestión (véase un ejemplo en la tabla 7).

10211. Definir qué es la tecnología puede tener dificultad, porque ésta sirve para muchas cosas. Pero la tecnología PRINCIPALMENTE es:

A. Muy parecida a la ciencia.

B. La aplicación de la ciencia.

C. Nuevos procesos, instrumentos, maquinaria, herramientas, aplicaciones, artilugios, ordenadores y aparatos prácticos para el uso de cada día.

D. Robots, electrónica, ordenadores, sistemas de comunicación y automatismos.

E. Una técnica para construir cosas o una forma de resolver problemas prácticos.

F. Inventar, diseñar y probar cosas (por ejemplo, corazones artificiales, ordenadores y vehículos espaciales).

G. Ideas y técnicas para diseñar y hacer cosas, para organizar a los trabajadores, la gente de negocios y los consumidores, y para el progreso de la sociedad.

H. Saber cómo hacer cosas (por ejemplo, instrumentos, maquinaria y aparatos).

Tabla 7.- Ejemplo de una cuestión del COCTS.

Un aspecto a resaltar del COCTS es su construcción empírica a partir de la interpretación cualitativa de las respuestas a entrevistas y preguntas abiertas hechas a estudiantes y profesores, de modo que las frases incluidas en sus ítems sintetizan una gran diversidad de creencias respecto a cada cuestión. Las sucesivas mejoras realizadas en la metodología de aplicación del cuestionario son otro aspecto a destacar (Acevedo, Acevedo, Manassero y Vázquez, 2001; Manassero y Vázquez, 1998; Manassero, Vázquez y Acevedo, 2001, 2004; Vázquez, Acevedo y Manassero, 2000; Vázquez y Manassero, 1999; Vázquez, Manassero y Acevedo, 2005, 2006).

\section{Procedimiento}

Para evitar sesgos inducidos por una posible diferencia en la comprensión de la tarea a realizar, los jueces recibieron instrucciones escritas normalizadas explicándoles el objetivo de la investigación y lo que tenían que hacer. Los jueces valoraron la adecuación de cada una de las frases del COCTS, en el contexto de cada cuestión y desde la perspectiva de los conocimientos de historia, filosofía y sociología de la ciencia, utilizando para ello una escala de nueve puntos (1-9), cuyas puntuaciones tienen el significado que se indica en la tabla 8.

La asignación por un juez de una puntuación alta a una frase significa que está de acuerdo con que ésta refleja una creencia adecuada, mientras que las puntuaciones más bajas significan que la frase representa una creencia ingenua o inadecuada 
respecto a la cuestión planteada. Los nueve valores de la escala se dividieron en tres intervalos cuyos significados, por la definición explícita de la escala, se corresponden con las tres categorías siguientes:

- Puntuaciones 1 a 3: categoría de frases ingenuas o inadecuadas.

- Puntuaciones 4 a 6: categoría de frases plausibles o parcialmente aceptables.

- Puntuaciones 7 a 9: categoría de frases adecuadas o apropiadas.

\begin{tabular}{|c|c|c|c|c|c|c|c|c|}
\hline \multicolumn{6}{|c|}{$\leftarrow$ MENOS ADECUADAS } & \multicolumn{3}{|c|}{ MÁS ADECUADAS $\rightarrow$} \\
\hline \multicolumn{3}{|c|}{ Ingenuas, inadecuadas } & \multicolumn{3}{|c|}{$\begin{array}{c}\text { Plausibles, parcialmente } \\
\text { aceptables }\end{array}$} & \multicolumn{3}{|c|}{ Adecuadas, apropiadas } \\
\hline $\begin{array}{l}\text { Totalme } \\
\text { nte } \\
\text { ingenuas }\end{array}$ & $\begin{array}{l}\text { Bastante } \\
\text { ingenuas }\end{array}$ & Ingenuas & $\begin{array}{c}\text { Poco } \\
\text { plausibles }\end{array}$ & Plausibles & $\begin{array}{l}\text { Bastante } \\
\text { plausibles }\end{array}$ & Adecuadas & $\begin{array}{l}\text { Bastante } \\
\text { adecuadas }\end{array}$ & $\begin{array}{l}\text { Totalmente } \\
\text { adecuadas }\end{array}$ \\
\hline 1 & 2 & 3 & 4 & 5 & 6 & 7 & 8 & 9 \\
\hline
\end{tabular}

Tabla 8.- Escala de valoración de cada frase del COCTS con la interpretación de su significado ${ }^{3}$.

Estas asignaciones definen intervalos naturales de puntuaciones con un significado fijo en la escala. Los intervalos de puntuaciones corresponden a distinto grado de acuerdo con las frases ingenuas en el rango 1 a 3 , del mismo modo que las puntuaciones entre 4 y 6 lo indican para las frases plausibles y las puntuaciones entre 7 y 9 lo hacen para las frases adecuadas (Acevedo, Acevedo, Manassero y Vázquez, 2001; Manassero, Vázquez y Acevedo, 2001; Vázquez, Acevedo y Manassero, 2000).

Las puntuaciones directas otorgadas por cada juez a cada frase se corresponden biunívocamente con su carácter ingenuo $(1,2,3)$, plausible $(4,5,6)$ o adecuado $(7$, $8,9)$, de modo que se pueden considerar como si fueran votos a favor de la categoría asociada a la puntuación emitida. Si una mayoría cualificada de dos tercios de los jueces (11 sobre 16) coincide asignando una puntuación de la categoría adecuada (7, 8 ó 9) a una frase, se interpreta que hay consenso respecto a que se trata de una creencia adecuada sobre NdC. Si la misma mayoría de dos tercios coincide dando una puntuación de la categoría ingenua ( 1,2 ó 3) a una frase, se considera que hay consenso respecto a que se trata de una creencia inadecuada o ingenua sobre $\mathrm{NdC}$.

\section{CONCLUSIÓN}

La metodología expuesta en este trabajo permite establecer empíricamente consensos respecto a la $\mathrm{NdC}$, entendida ésta en un sentido amplio que no se limita a la dimensión epistemológica sino que se extiende a otras relacionadas con la sociología interna y la sociología externa de la ciencia, en coherencia con las dimensiones del COCTS.

\footnotetext{
${ }^{3}$ Ingenua: la frase expresa una creencia que no es ni apropiada ni plausible. Plausible: aunque no es completamente adecuada, la frase expresa algunos aspectos apropiados desde la perspectiva de los conocimientos de historia, filosofía y sociología de la ciencia. Adecuada: la frase expresa una creencia apropiada desde la perspectiva de los conocimientos de historia, filosofía y sociología de la ciencia.
} 
Como se ha señalado en este artículo, algunos estudios precedentes se han centrado específicamente en el consenso respecto a creencias adecuadas (Eflin, Glennan y Reisch, 1999; Felske, Chiappetta y Kemper, 2001; Lederman, Abd-El-Khalick, Bell y Schwartz, 2002; McComas, Clough y Almazroa, 1998; McComas y Olson, 1998; Osborne et al., 2003), o sobre creencias ingenuas (Fernández et al., 2002, 2003; Fernández, Gil-Pérez, Valdés y Vilches, 2005; Gil-Pérez et al., 2005; McComas, 1996, 1998), pero no en ambos a la vez. Por el contrario, los acuerdos que se pueden alcanzar con el procedimiento expuesto se refieren de modo integrado, con una misma metodología y criterio, tanto a las creencias de $\mathrm{NdC}$ que se consideran adecuadas como a lo que se reconocen inadecuadas, necesarias ambas para ser tratadas en la enseñanza de las ciencias, lo que supondrá un avance en la fundamentación empírica de las mismas.

La presentación simultánea de creencias adecuadas e ingenuas relativas a las diversas cuestiones del COCTS constituye, asimismo, una propuesta innovadora de esta investigación para la didáctica de las ciencias. En efecto, en cuestiones tan controvertidas como las propias de $\mathrm{NdC}$, no sólo son importantes las afirmaciones en positivo sino también en negativo, pues ambas contribuyen a mejorar el aprendizaje. La enseñanza de los rasgos positivos junto con los rasgos negativos puede contribuir a precisar mejor los contenidos más difíciles y escurridizos de la $\mathrm{NdC}$, por lo que ambos tipos de consensos deberían tratarse de modo explícito como contenidos en el currículo de ciencias. Una parte de los consensos alcanzados ya ha sido anticipada (Vázquez, Acevedo y Manassero, 2004, 2005; Vázquez, Acevedo, Manassero y Acevedo, 2004, 2005, 2006) y todos los acuerdos se expondrán completamente y se discutirán con suficiente detalle en próximos trabajos, en esta misma revista (Acevedo, Vázquez, Manassero y Acevedo, 2007 en prensa) y en otras (Vázquez, Acevedo, Manassero y Acevedo, 2007 en prensa; Vázquez, Manassero, Acevedo y Acevedo, 2007 en prensa).

\section{REFERENCIAS}

AAAS American Association for the Advancement of Science (1989). Project 2061: Science for all Americans. Washington, DC: American Association for the Advancement of Science.

AAAS American Association for the Advancement of Science (1993). Project 2061. Benchmarks for science literacy. New York, NY: Oxford University Press.

ACEVEDO, J. A. (1997). ¿Publicar o patentar? Hacia una ciencia cada vez más ligada a la tecnología. Revista Española de Física, 11(2), 8-11. Consultado 16/9/2006 en Sala de Lecturas CTS+I de la OEI, 2001, http://www.campusoei.org/salactsi/acevedo4.htm.

ACEVEDO, J. A. (1998). Análisis de algunos criterios para diferenciar entre ciencia y tecnología. Enseñanza de las Ciencias, 16(3), 409-420.

ACEVEDO, J. A. (2005). TIMSS Y PISA. Dos proyectos internacionales de evaluación del aprendizaje escolar en ciencias. Revista Eureka sobre Enseñanza y Divulgación de las Ciencias, 2(3), 282-301. Consultado 16/9/2006 en http://www.apac-eureka.org/revista/Larevista.htm. 
ACEVEDO, J. A. (2006). Modelos de relaciones entre ciencia y tecnología: un análisis social e histórico. Revista Eureka sobre Enseñanza y Divulgación de las Ciencias, 3(2), 198-219. Consultado 16/9/2006 en http://www.apaceureka.org/revista/Larevista.htm.

ACeVedo, J. A., ACeVedo, P., MANASSero, M. A. y VÁzQuez, A. (2001). Avances metodológicos en la investigación sobre evaluación de actitudes y creencias CTS. Revista Iberoamericana de Educación, edición electrónica. O.E.I. Consultado 15/9/2006 en: http://www.campusoei.org/revista/deloslectores/Acevedo.PDF.

ACEVEDO, J. A., MANASSERO, M. A. y VÁZQUEZ, A. (2002). Nuevos retos educativos: Hacia una orientación CTS de la alfabetización científica y tecnológica. Revista Pensamiento Educativo, 30, 15-34.

ACEVEDO, J. A., MANASSERO, M. A. y VÁZQUEZ, A. (2005). Orientación CTS de la alfabetización científica y tecnológica de la ciudadanía: un desafío educativo para el siglo XXI. En P. Membiela e Y. Padilla (Eds.), Retos y perspectivas de la enseñanza de las ciencias desde el enfoque CTS en los inicios del siglo XXI (pp. 7-14). Vigo: Educación Editora. Consultado 15/9/2006 en http://webs.uvigo.es/educacion.editora/.

ACEVEDO, J. A., VÁZQUEZ, A. y MANASSERO, M. A. (2002). El movimiento Ciencia, Tecnología y Sociedad y la enseñanza de las ciencias. Consultado 15/9/2006 en Sala de Lecturas CTS $+I$ de la OEI, http://www.campusoei.org/salactsi/acevedo13.htm. Versión en castellano del capítulo 1 del libro de Manassero, M. A., Vázquez, A. y Acevedo, J. A. (2001): Avaluació dels temes de ciència, tecnologia i societat. Palma de Mallorca: Conselleria d'Educació i Cultura del Govern de les Illes Ballears.

ACEVEdo, J. A., VÁZQUeZ, A. y MANASSERO, M. A. (2003). Papel de la educación CTS en una alfabetización científica y tecnológica para todas las personas. Revista Electrónica de Enseñanza de las Ciencias, 2(2). Consultado 15/9/2006 en http://www.saum.uvigo.es/reec/.

ACEVEDO, J. A., VÁZQUeZ, A., MANASSERO, M. A. y ACEVEDO, P. (2007, en prensa). Consensos sobre la naturaleza de la ciencia: aspectos epistemológicos. Revista Eureka sobre Enseñanza y Divulgación de las Ciencias, 4(2).

AIKENHEAD, G. S. (2003). STS Education: A Rose by Any Other Name. En R. T. Cross (Ed.), A Vision for Science Education: Responding to the work of Peter J. Fensham (pp. 59-75). New York, NY: Routledge Falmer. Consultado 15/9/2006 en http://www.usask.ca/education/people/aikenhead/stsed.htm.

AIKENHEAD, G. S. y FLEMING, R. (1975). Science: A way of knowing. Saskatoon, Saskatchewan, Canada: University of Saskatchewan, Department of Curriculum Studies.

AIKENHEAD, G. S. y RYAN, A. G. (1989). The development of a multiple choice instrument for monitoring views on Science-Technology-Society topics. Final Report of SSHRCC Grant: Author.

AIKENHEAD, G. S. y RYAN, A. G. (1992). The development of a new instrument: "Views on science-technology-society" (VOSTS). Science Education, 76(5), 477-491. 
AIKENHEAD, G. S., RYAN, A. S. y FLEMING, R. W. (1989). Views on sciencetechnology-society (form CDN. mc. 5). Saskatoon, Canadá: Department of Curriculum Studies, College of Education, University of Saskatchewan. Consultado 15/9/2006

en http://www.usask.ca/education/people/aikenhead/vosts.pdf.

ALTERS, B. J. (1997). Whose nature of science? Journal of Research in Science Teaching, 34(1), 39-55.

BARTHOLOMEW, H., OSBORNE, J. y RATCLIFFE, M. (2004). Teaching Students "IdeasAbout-Science": Five Dimensions of Effective Practice. Science Education, 88(5), 655-682.

BELL, R. L. (2005). The Nature of Science in Instruction and Learning. Paper presented at the Annual International Conference of the Association for the Education of Teachers in Science (AETS), Colorado Springs, CO.

BELL, R. L., ABD-EL-KHALICK, F., LEDERMAN, N. G., McCOMAS, W. F. y MATTHEWS, M. R. (2001). The Nature of Science and Science Education: A Bibliography. Science \& Education, 10(1/2), 187-204.

COLLINS, H. M. (1985). Changing Order: Replication and Induction in Scientific Practice. London: Sage. $2^{\text {nd }}$ edition (1992). Chicago, IL: University of Chicago Press].

DRIVER, R., LEACH, J., MILLAR, R. y SCOTT, P. (1996). Young people's images of science. Buckingham: Open University Press.

EAGLY, A. H. y CHAIKEN, S. (1993). The psychology of attitudes. Forth Worth: Harcourt Brace College Publishers.

ECHEVERRÍA, J. (1999). Introducción a la metodología de la ciencia: la filosofía de la ciencia en el siglo $X X$. Madrid: Cátedra.

ECHEVERRÍA, J. (2002). Ciencia y valores. Barcelona: Destino.

EFLIN, J. T., GLENNAN, S. y REISCH, R. (1999). The Nature of Science: A Perspective from the Philosophy of Science. Journal of Research in Science Teaching, 36, (1), 107-116.

FELSKE, D. D., CHIAPPETTA, E. y KEMPER, J. (2001). A Historical Examination of the Nature of Science and its Consensus in Benchmarks and Standards. Paper presented at the Annual Meeting of the National Association for Research in Science Teaching. St. Louis, MO.

FERNÁNDEZ, I., GIL, D., CARRASCOSA, J., CACHAPUZ, A. y PRAIA, J. (2002). Visiones deformadas de la ciencia transmitidas por la enseñanza. Enseñanza de las Ciencias, 20(3), 477-488.

FERNÁNDEZ, I., GIL-PÉREZ, D., VALDÉS, P. y VILCHES, A. (2005). ¿Qué visiones de la ciencia y la actividad científica tenemos y transmitimos? En D. Gil-Pérez, B. Macedo, J. Martínez-Torregrosa, C. Sifredo, P. Valdés y A. Vilches (Eds.), ¿Cómo promover el interés por la cultura científica? Una propuesta didáctica fundamentada para la educación científica de jóvenes de 15 a 18 años (pp. 2962). Santiago, Chile: OREALC/UNESCO.

FERNÁNDEZ, I., GIL, D., VILCHES, A., VALDÉS, P., CACHAPUZ, A., PRAIA, J. y SALINAS J. (2003). El olvido de la tecnología como refuerzo de las visiones deformadas de la ciencia. Revista Electrónica de Enseñanza de las Ciencias, 2(3). Consultado 16/9/2006 en http://www.saum.uvigo.es/reec/. 
GIL-PÉREZ, D., VILCHES, A., FERNÁNDEZ, I., CACHAPUZ, A., PRAIA, J., VALDÉS, P. y SALINAS, J. (2005). Technology as 'Applied Science': A Serious Misconception that Reinforces Distorted and Impoverished Views of Science. Science \& Education, 14 (3-5), 309-320.

GROSS, P. y LEVITT, N. (1994). Higher superstition: The academic left and its quarrels with science. Baltimore: John Hopkins University Press.

HODSON, D. (1988). Toward a philosophically more valid science curriculum. Science Education, 72(1), 19-40.

LAUDAN, L. (1990). Science and relativism: Some key controversies in the philosophy of science. Chicago, IL: University of Chicago Press. [(1993) La ciencia y el relativismo. Controversias básicas en filosofía de la Ciencia, Madrid: Alianza Editorial].

LAUDAN, L. (1996). Beyond Positivism and Relativism. Theory, Method and Evidence. Oxford: Westview Press.

LEDERMAN, N. G. (1992). Students' and teachers' conceptions of the nature of science: A review of the research. Journal of Research in Science Teaching, 29(4), 331-359.

LEDERMAN, N. G. (1999). Teachers' understanding of the nature of science: Factors that facilitate or impede the relationship. Journal of Research in Science Teaching, 36(8), 916-929.

LEDERMAN, N., ABD-EL-KHALICK, F., BELL, R. L. y SCHWARTZ, R. S. (2002). Views of Nature of Science Questionnaire: Towards valid and meaningful assessment of learners' conceptions of the nature of science. Journal of Research in Science Teaching, 39(6), 497-521.

LEDERMAN, N. G., WADE, P. D. y BELL, R. L. (1998). Assessing understanding of the nature of science: A historical perspective. En W. F. McComas (Ed.), The nature of science in science education: Rationales and strategies (pp. 331-350). Dordrecht, The Netherlands: Kluwer Academic Publishers.

LONGINO, H. E. (1983). Beyond "bad science": Skeptical reflections on the valuefreedom of scientific inquiry. Science, Technology, and Human Values, 8(1), 717.

LONGINO, H. E. (1990). Science as social knowledge: Values and objectivity in scientific inquiry. Princeton, NJ: Princeton University Press.

MANASSERO, M. A. Y VÁZQUEZ, A. (1998). Opinions sobre ciència, tecnologia $i$ societat. Palma de Mallorca: Conselleria d'Educació, Cultura i Esports del Govern de les Illes Ballears.

MANASSERO, M. A., VÁZQUEZ, A. y ACEVEDO, J. A. (2001). Avaluació del temes de ciència, tecnologia $i$ societat. Palma de Mallorca: Conselleria d'Educació i Cultura del Govern de les Illes Ballears.

MANASSERO, M. A., VÁZQUEZ, A. y ACEVEDO, J. A. (2003). Cuestionario de Opiniones sobre Ciencia, Tecnología y Sociedad (COCTS). Princeton, NJ: Educational Testing Service. Consultado 14/9/2006 en http://www.ets.org/testcoll/.

MANASSERO, M. A., VÁZQUEZ, A. Y ACEVEDO, J. A. (2004). Evaluación de las actitudes del profesorado respecto a los temas CTS: nuevos avances metodológicos. Enseñanza de las Ciencias, 22(2), 299-312. 
MATTHEWS, M. R. (1994). Science teaching: The role of history and philosophy of science. New York: Routledge.

McCOMAS, W. F. (1996). Ten Myths of Science: reexamining what we think we know about the nature of science. School Science and Mathematics, 96(1), 10-16.

McCOMAS, W. F. (1998). The principal elements of the nature of science: Dispelling the myths. En W. F. McComas. (Ed.), The Nature of Science in Science Education (pp. 53-72). Dordrecht, The Netherlands: Kluwer Academic Publishers.

McCOMAS W. F., CLOUGH, M. P. y ALMAZROA, H. (1998). The Role and Character of the Nature of Science in Science Education. En W. F. McComas (Ed.), The Nature Of Science In Science Education. Rationales and Strategies (pp. 3-39). Dordrecht, The Netherlands: Kluwer Academic Publishers.

McCOMAS, W. F. y OLSON, J. K. (1998). The nature of science in international science education standards documents. En W. F. McComas (Ed.), The nature of science in science education: Rationales and strategies (pp. 41-52). Dordrecht, The Netherlands: Kluwer Academic Publishers.

MILLAR, R. (1996). Towards a science curriculum for public understanding. School Science Review, 77, 7-18.

MILLAR, R. y OSBORNE, J. (Eds.) (1998). Beyond 2000: Science education for the future. London: King's College London School of Education.

NORRIS, C. (1997). Against relativism: Philosophy of science, deconstruction and critical theory. Malden, MA: Blackwell.

NRC, National Science Teachers Association (1996). National Science Education Standards. Washington DC: National Academy Press.

OECD (2003). The PISA 2003 Assessment Framework: Mathematics, Reading, Science and Problem Solving Knowledge and Skills. París: OECD. Traducción de E. Belmonte (2004): Marcos teóricos de PISA 2003: la medida de los conocimientos y destrezas en matemáticas, lectura, ciencias y resolución de problemas. Madrid: INECSE/MEC.

OGBORN, J., KRESS, G., MARTINS, I. y McGILLICUDDY, K. (1996). Explaining science in the classroom. Buckingham: Open University Press.

OSBORNE, J., COLLINS, S., RATCLIFFE, M., MILLAR, R. y DUSCHL, R. (2003). What "Ideas-about-Science" Should Be Taught in School Science? A Delphi Study of the Expert Community. Journal of Research in Science Teaching, 40(7), 692720.

RUBBA, P. A. y HARKNESS, W. L. (1993). Examination of preservice and in-service secondary science teachers' beliefs about Science-Technology-Society interactions. Science Education, 77(4), 407-431.

RUBBA, P. A., SCHONEWEG-BRADFORD, C. y HARKNESS, W. L. (1996). A new scoring procedure for the Views on Science-Technology-Society instrument. International Journal of Science Education, 18(4), 387-400.

RUDOLPH, J. L. (2003). Portraying epistemology: School science in historical context. Science Education, 87(1), 64-79.

RYAN, A. G., y AIKENHEAD, G. S. (1992). Students' preconceptions about the epistemology of science. Science Education, 76 (6), 559-580. 
RYDER, J., LEACH, J., y DRIVER, R. (1999). Undergraduate science students' images of science. Journal of Research in Science Teaching, 36(2), 201-220.

SMITH, M. U., LEDERMAN, N. G., BELL, R. L., McCOMAS, W. F. y CLOUGH, M. P. (1997). How great is the disagreement about the nature of science? A response to Alters. Journal of Research in Science Teaching, 34(10), 1101-1104.

SMITH, M. U. y SCHARMANN, L. C. (1999). Defining versus describing the nature of science: A pragmatic analysis for classroom teachers and science educators. Science Education, 83(4), 493-509.

SOKAL, A. (1996). Transgressing the boundaries: towards a transformative hermeneutics of quantum gravity. Social Text, 46/47, 217-252.

SOKAL, A. y BRICMONT, J. (1998). Intellectual impostures: Postmodern philosophers' abuse of science. London: Profile. [(1999) Imposturas intelectuales, Barcelona: Paidós].

SPECTOR, B., STRONG, P. y LAPORTA, T. (1998). Teaching the nature of science as an element of science, technology and society. En W. F. McComas (Ed.), The nature of science in science education. Rationales and strategies (pp. 267-276). Dordrecht, The Netherlands: Kluwer Academic Publishers.

VÁzQUEZ, A., ACEVEDO, J. A. y MANASSERO, M. A. (2000). Progresos en la evaluación de actitudes relacionadas con la ciencia mediante el Cuestionario de Opiniones CTS. En I. P. Martins (Org.), O Movimento CTS na Península Ibérica. Seminário Ibérico sobre Ciência-Tecnologia-Sociedade no ensino-aprendizagem das ciencias experimentais (pp. 219-230). Aveiro: Universidade de Aveiro. Consultado 16/9/2006 en Sala de Lecturas CTS+I de la OEI, 2001, http://www.campus-oei.org/salactsi/acevedo6.htm.

VÁZQUEZ, A., ACEVEDO, J. A. y MANASSERO, M. A. (2004). Consensos sobre la naturaleza de la ciencia: evidencias e implicaciones para su enseñanza. Revista Iberoamericana de Educación, edición electrónica. Consultado 15/9/2006 en http://www.campus-oei.org/revista/deloslectores/702Vazquez.PDF.

VÁZQUEZ, A., ACEVEDO, J. A. y MANASSERO, M. A. (2005). The dark side of the nature of science: empirical consensus about naïve ideas on science. Paper presented at the $5^{\text {th }}$ Conference of the European Science Education Research Association (ESERA): Contributions of Research to Enhancing Students' Interest in Learning Science. Barcelona, Spain (28 August - 1 September, 2005).

VÁzQUez, A., ACEVEdo, J. A., MANASSERO, M. A. y ACEVEdo, P. (2001). Cuatro paradigmas básicos sobre la naturaleza de la ciencia. Argumentos de Razón Técnica, 4, 135-176. Consultado 15/9/2006 en Sala de Lecturas CTS+I de la OEI, 2003, http://www.campus-oei.org/salactsi/acevedo20.htm.

VÁZQUEZ, A., ACEVEDO, J. A., MANASSERO, M. A. y ACEVEDO, P. (2004). Hacia un consenso sobre la naturaleza de la ciencia en la enseñanza de las ciencias. En I. P. Martins, F. Paixão y R. Vieira (Org.): Perspectivas Ciência-Tecnologia-Sociedade na Inovação da Educação em Ciência. Aveiro (Portugal): Universidade de Aveiro, pp. 129-132.

VÁZQUeZ, A., ACEVEDO, J. A., MANASSERO, M. A. y ACEVEDO, P. (2005). Consensos sobre la naturaleza de la ciencia para la enseñanza de las ciencias. Comunicación presentada en el VII Congreso Internacional sobre Investigación en la Didáctica de las Ciencias: Educación científica para la ciudadanía 
(Granada, 7-10 de septiembre de 2005). Enseñanza de las Ciencias, no extra (VII Congreso), edición en CD. Consultado 16/9/2006 en http://blues.uab.es/rev-ens-ciencias/.

VÁzQUeZ, A., ACEVEdo, J. A., MANASSERo, M. A., y ACEVEdo, P. (2006). Creencias ingenuas sobre naturaleza de la ciencia: consensos en sociología interna de ciencia y tecnología. Actas del IV Seminario Ibérico de CTS en la Educación Científica: Las relaciones CTS en la Educación Científica. Málaga: Universidad de Málaga (3-5 de julio de 2006), edición en CD.

VÁzQUeZ, A., ACEVEdo, J. A., MANASSERO, M. A. y ACEVEDO, P. (2007 en prensa). Consensos sobre la naturaleza de la ciencia: la ciencia y la tecnología en la sociedad. Enviado a publicación.

VÁZQUEZ, A. y MANASSERO, M. A. (1995). Actitudes relacionadas con la ciencia: una revisión conceptual. Enseñanza de las Ciencias, 13, 337-346.

VÁZQUEZ, A. y MANASSERO, M. A. (1999). New response and scoring models for the "Views on Science-Technology-Society" instrument (VOSTS). International Journal of Science Education, 21(3), 231-247.

VÁZQUEZ, A., MANASSERO, M. A. y ACEVEDO, J. A. (2005). Análisis cuantitativo de ítems complejos de opción múltiple en ciencia, tecnología y sociedad: Escalamiento de ítems. Revista Electrónica de Investigación Educativa, 7(1). Consultado 16/9/2006 en http://redie.uabc.mx/vol7no1/contenidovazquez.html.

VÁZQUeZ, A., MANASSERO, M. A. y ACEVEDO, J. A. (2006). An Analysis of Complex Multiple-Choice Science-Technology-Society Items: Methodological Development and Preliminary Results. Science Education, 90(4), 681-706.

VÁZQUeZ, A., MANASSERO, M. A., ACEVEDO, J. A. y ACEVEDO, P. (2007 en prensa). Consensos sobre la naturaleza de la ciencia: la comunidad tecnocientífica. Educación en Química, 18(1).

WOLPERT, L. (1992). The Unnatural Nature of Science. London: Faber y Faber. [(1994) La naturaleza no natural de la ciencia, Madrid: Acento Editorial].

ZIMAN, J. (2000). Real science: What it is and what it means. Cambridge: Cambridge University Press. [(2003) ¿Qué es la ciencia? Madrid: Cambridge University Press].

ZIMAN, J. (2003). Ciencia y sociedad civil. Revista Iberoamericana de Ciencia, Tecnología y Sociedad, 1(1), 177-188. Publicado también el mismo año en Isegoría, 28, 5-17. 


\section{SUMMARY}

The issues about the nature of science are today considered a core content of the scientific and technological literacy for all. However, the inclusion of the nature of science in the science school curriculum is not easy and requires some conditions. One important condition is setting up previous agreements on the specific features and contents of the nature of science that could be translated into the classroom practice for its explicit use in school science education. This paper reviews some contributions of the science education research to achieve this condition. Finally, an empiric methodology designed to achieve consensus on the nature of science is put forward; the description embraces the agreements on the appropriate as well as on the inappropriate features of the nature of science.

Keywords: Nature of the science, disagreement, consensus, science school curriculum, Questionnaire of Opinions on STS, empiric methodology. 